\title{
Alpha-synuclein oligomers and small nerve fiber pathology in skin are potential biomarkers of Parkinson's disease
}

\author{
Elena Vacchi $\mathbb{D}^{1,2}$, Camilla Senese ${ }^{1}$, Giacomo Chiaro ${ }^{3}$, Giulio Disanto ${ }^{3}$, Sandra Pinton ${ }^{1}$, Sara Morandi ${ }^{3}$, llaria Bertaina ${ }^{3}$, Giovanni Bianco ${ }^{3}$, \\ Claudio Staedler ${ }^{3}$, Salvatore Galati ${ }^{2,3}$, Claudio Gobbi ${ }^{2,3}$, Alain Kaelin-Lang ${ }^{1,2,3,4}$ and Giorgia Melli $\mathbb{1}^{1,2,3 凶}$
}

The proximity ligation assay (PLA) is a specific and sensitive technique for the detection of aSyn oligomers (aSyn-PLA), early and toxic species implicated in the pathogenesis of PD. We aimed to evaluate by skin biopsy the diagnostic and prognostic capacity of aSyn-PLA and small nerve fiber reduction in PD in a longitudinal study. aSyn-PLA was performed in the ankle and cervical skin biopsies of PD $(n=30)$, atypical parkinsonisms (AP, $n=23$ ) including multiple system atrophy (MSA, $n=12$ ) and tauopathies (APTau, $n=11$ ), and healthy controls (HC, $n=22$ ). Skin biopsy was also analyzed for phosphorylated aSyn (P-aSyn) and 5G4 (aSyn5G4), a conformation-specific antibody to aggregated aSyn. Intraepidermal nerve fiber density (IENFD) was assessed as a measure of small fiber neuropathy. aSyn-PLA signal was more expressed in PD and MSA compared to controls and AP-Tau. aSyn-PLA showed the highest diagnostic accuracy (PD vs. HC sensitivity $80 \%$, specificity $77 \%$; PD vs. AP-Tau sensitivity $80 \%$, specificity $82 \%$ ), however, P-aSyn and 5G4, possible markers of later phases, performed better when considering the ankle site alone. A small fiber neuropathy was detected in PD and MSA. A progression of denervation not of pathological aSyn was detected at follow-up and a lower IENFD at baseline was associated with a greater cognitive and motor decline in PD. A skin biopsy-derived compound marker, resulting from a linear discrimination analysis model of aSyn-PLA, P-aSyn, aSyn-5G4, and IENFD, stratified patients with accuracy (77.8\%), including the discrimination between PD and MSA (84.6\%). In conclusion, the choice of pathological aSyn marker and anatomical site influences the diagnostic performance of skin biopsy and can help in understanding the temporal dynamics of aSyn spreading in the peripheral nervous system during the disease. Skin denervation, not pathological aSyn is a potential progression marker for PD.

npj Parkinson's Disease (2021)7:119; https://doi.org/10.1038/s41531-021-00262-y

\section{INTRODUCTION}

Parkinson's disease (PD) is a neurodegenerative disease characterized by the presence of pathologic alpha-synuclein (aSyn) deposits in specific areas of the brain. In association with dementia with Lewy bodies (DLB) and multiple system atrophy (MSA), it is part of a spectrum of disorders, the synucleinopathies, characterized by intraneuronal aggregates of aSyn called Lewy bodies and Lewy neurites (PD and DLB) and by glial cytoplasmatic inclusions of aSyn (MSA) ${ }^{1,2}$.

PD is a multisystem disorder that involves both the central and peripheral nervous system ${ }^{3}$. Indeed, while the overt phenotype of $\mathrm{PD}$ is that of a movement disorder characterized by bradykinesia in combination with at least one of rest tremor, rigidity, or postural instability, several non-motor autonomic symptoms affect PD patients in prodromal phases ${ }^{4}$. Typical examples are urinary and gastrointestinal dysfunction, impaired sudomotor function, and orthostatic hypotension. Accordingly, in the last decade, multiple studies have shown evidence of aSyn deposits in peripheral nerve tissues and organs, opening the possibility to access pathological tissue in a minimally invasive way ${ }^{5-7}$. This is of utter relevance, considering that the lack of antemortem biomarkers is a major limiting factor for developing specific and effective diseasemodifying therapies for neurodegenerative disorders. Furthermore, the clinical heterogeneity of PD and the overlapping clinical spectrum with atypical parkinsonisms (AP), namely MSA and AP with tauopathies (AP-Tau), often prevent a proper diagnosis and management of patients, especially in early phases ${ }^{8}$. In particular, aSyn phosphorylated at Serine 129 (P-aSyn) has been detected in colonic mucosa ${ }^{9}$, submandibular glands ${ }^{10}$, skin $^{11-14}$, and heart ${ }^{15}$ of patients with PD. More recently 5G4 antibody, a conformationspecific monoclonal antibody with high reactivity for aggregated forms of aSyn, and low reactivity for monomeric aSyn ${ }^{16-18}$, has been successfully exploited in the skin ${ }^{14}$, submandibular glands ${ }^{19}$, and colonic mucosa ${ }^{20}$. A systematic meta-analysis on in vivo aSyn detection in peripheral tissues has demonstrated that skin biopsy examination using anti-P-aSyn antibody has the best diagnostic accuracy for $\mathrm{PD}^{21}$. Moreover, through intraepidermal nerve fiber density (IENFD) measurement, skin biopsy allowed to demonstrate and quantify small fiber neuropathy in $P D^{14,22}$, which confirms the observation of corneal small nerve fiber reduction in confocal microscopy ${ }^{23}$. However, discrepancies of reported specificity and sensitivity, diverse sites of skin biopsy, and lack of standardized protocols, are major issues in using skin biopsy as a definitive diagnostic tool for PD.

In this study, we exploited proximity ligation assay (PLA), a highly sensitive and specific technique for oligomeric aSyn detection (aSyn-PLA), in skin biopsies of PD in a longitudinal study. PLA is based on proximity probes, composed of oligonucleotide-conjugated antibodies, to recognize a couple of specific targets in close proximity $(<16 \mathrm{~nm})^{24}$. aSyn-PLA assay exploits a monoclonal antibody to aSyn with blocking activity on the epitope and preferentially targets aSyn oligomers compared to monomers and fibrils in vitro and binds to early not compacted

\footnotetext{
'Laboratory for Biomedical Neurosciences, Neurocenter of Southern Switzerland, Ente Ospedaliero Cantonale, Lugano, Switzerland. ${ }^{2}$ Faculty of Biomedical Sciences, Università della Svizzera Italiana, Lugano, Switzerland. ${ }^{3}$ Neurology Department, Neurocenter of Southern Switzerland, Ente Ospedaliero Cantonale, Lugano, Switzerland. ${ }^{4}$ Department of Neurology, Inselspital, Bern University Hospital, University of Bern, Bern, Switzerland. ${ }^{凶}$ email: giorgia.melli@eoc.ch
} 
lesions such us pale bodies, and only rarely to late compacted Lewy bodies in patients' brain ${ }^{25}$. This is of relevance since aSyn oligomers are early and toxic species implicated in the pathogenesis and spread of disease ${ }^{26}$. The presence of aSyn oligomers was previously detected by aSyn-PLA in the brain of postmortem PD ${ }^{25}$ and $\mathrm{MSA}^{27}$, in in vivo gastrointestinal tissues ${ }^{28}$, and very recently, in skin biopsies of PD patients ${ }^{29}$. In particular, Mazzetti et al. described aSyn-PLA within synaptic terminals of cutaneous autonomic fibers in PD and healthy controls $(\mathrm{HC})$, observing a major expression of oligomeric aSyn in patients ${ }^{29}$.

This study aimed to (1) analyze oligomeric aSyn in the ankle and cervical skin biopsies of patients with PD, MSA, AP-Tau, and HC; (2) compare the diagnostic accuracy for PD of aSyn-PLA vs. P-aSyn and vs. 5G4 antibody according to the anatomical site; (3) evaluate pathologic aSyn and IENFD changes by skin biopsy longitudinally at 2 years follow-up in PD.

\section{RESULTS}

\section{Demographics and clinical data}

Demographic characteristics and clinical assessments of the study groups are summarized in Table 1. As expected, AP patients showed a more severe disease than PD, measured by H\&Y and MDS-UPDRS, and a higher cognitive impairment, measured by MMSE and MoCA. AP-Tau subjects were significantly older than HC and more depressed than PD. MSA showed a greater autonomic dysfunction measured by COMPASS- 31 .

After 24 months, 6 patients died (one PD, two MSA, and three AP-Tau), 18 patients dropped out of the study (five PD, five MSA, and eight AP-Tau). Twenty-four of the 30 PD patients underwent a 24-month follow-up analysis (T24) (Fig. 1). The six PD patients who did not perform the T24 assessment were significantly more affected than the rest of the PD group (H\&Y, $P=0.050$; MDSUPDRS-III, $P=0.031$ ) (Supp. Table 1). Since few subjects with MSA and none with AP-Tau performed the T24 evaluation, we did not perform statistical analysis at T24 in these groups.

\section{PD and MSA display a small nerve fiber pathology}

PD and MSA showed a significant reduction of total and ankle IENFD compared to HC (Table 1). A tendency towards a reduced IENFD also at the cervical site was present in PD, even if not significant, possibly due to a higher variability.

\section{PD and MSA show high positivity for aSyn-PLA}

The co-localization signal, defined as an aSyn-PLA signal within the PGP9.5 positive nerves (Fig. 2a), was the most frequent, while the dotted one, defined as the signal located in proximity to degenerated nerve fibers or interposed between nerve fiber breakpoints (Fig. 2a), was observed exclusively in PD and MSA, not in AP-Tau or HC (Fig. 3a). In the MSA group, the dotted signal was always observed in association with the co-localization signal, while in the PD group 3, 3\% of patients showed a dotted signal only (Sup. Table 2).

The percentage of patients showing aSyn-PLA was significantly higher in PD and MSA groups compared to HC and AP-Tau (Fig. 3a-c and Sup. Table 2). Considering the two anatomical sites separately, the cervical area allowed the differentiation between PD and HC and AP-Tau (Fig. 3b). No differences were observed between groups at the ankle site (Fig. 3c). MSA showed a higher positivity at the ankle than at the cervical area (Fig. $3 c$ ). A double positivity at both anatomical sites was observed only in PD and MSA groups (16.7 and $11.1 \%$ respectively).

The majority of aSyn-PLA was detected in nerve fibers surrounding autonomic structures. In 2 out of 30 PD patients, the signal was observed in dermal nerve bundles. Most of the signal was evident in SG innervation (Fig. 3d and Sup. Table 2): PD and MSA group displayed a greater positivity compared to $\mathrm{HC}$ and AP-Tau, considering both localization and the cervical site alone (Fig. 3e). No differences between groups in PLA signal were observed in MAP.

MSA displayed a greater area of aSyn-PLA signal within nerves compared to PD (Fig. 3g-i and Sup. Table 3), especially at the cervical site. No differences were observed in the number of aSynPLA signals (Sup. Table 3).

Finally, the presence of aSyn-PLA was associated with the diagnosis of PD and MSA by univariate logistic regression analysis (Table 2). PD showed higher odds ratios compared to MSA, except for aSyn-PLA in SG. Moreover, an association with PD diagnosis was observed considering aSyn-PLA signal at cervical site only.

\section{aSyn-PLA showed higher diagnostic accuracy for PD and MSA} than P-aSyn and aSyn-5G4

In addition to aSyn-PLA, subjects were investigated for P-aSyn and aSyn-5G4. All markers were more expressed in PD than HC and AP-Tau, but aSyn-PLA displayed higher sensitivity when considering both locations and the cervical area (Fig. $4 a-c$ and Sup. Table 6). Within groups, no differences were displayed between the three markers (Fig. 4d-f). The ROC curve analysis demonstrated that aSyn-PLA had the highest AUC when considering both locations or the cervical site $(4 \mathrm{~g}, \mathrm{~h}, \mathrm{j}, \mathrm{k})$. Nevertheless, at the ankle, only P-aSyn and aSyn-5G4 could discriminate between groups (Fig. 4i-l), even if with a lower AUC than aSyn-PLA in the cervical area. Finally, only aSyn-PLA allowed the discrimination between MSA and HC, considering both anatomical sites together (Fig. $4 \mathrm{~m}$ ).

\section{PD patients present a progression of denervation not of pathological aSyn at T24}

The comparison between subjects with PD at time point 0 (T0) and after 24 months (T24), showed a significant reduction in total IENFD, ankle IENFD, and cervical IENFD (Fig. 5a-c). No differences in the percentage of patients positive for aSyn-PLA, P-aSyn, and aSyn-5G4 were detected (Fig. 5d-f), but a slight reduction for all markers at the cervical site and an increase of aSyn-PLA signal in the ankle were noted. No significant changes in clinical scales and LEDD were detected (Supp. Table 7).

\section{IENFD is a progression marker for PD}

Comparisons of skin biopsy parameters at T0 according to the clinical state at T24 demonstrated that: (1) lower cervical IENFD was measured in those presenting a progression of cognitive decline at the MMSE scale and (2) lower ankle IENFD was observed in the group presenting a progression of motor impairment at the MDS-UPDRS-III scale (Fig. 5g, h). These results were confirmed by logistic regression, showing that low cervical IENFD was associated with an increased risk of developing a cognitive decline, while low ankle IENFD was associated with an increased risk of developing motor impairment at T24, after adjusting for age and LEDD (Fig. 5i-k).

\section{A skin biopsy-derived compound marker stratified subjects according to the clinical diagnosis}

A linear discriminant analysis model based on the IENFD and the expression of aSyn-PLA, P-aSyn, and aSyn-5G4 at both sites at T0, allowed the separation of subjects according to their clinical diagnosis (Fig. 6a). Patients were discriminated with $77.8 \%$ of accuracy: 26 out of 30 PD, and five out of nine MSA were correctly diagnosed, while the discrimination between HC and AP-Tau was more challenging (Fig. 6b). Subsequently, pairwise comparisons were performed (Fig. 6c-h), showing high sensitivity (77.7-93.3\%) and specificity $(66.6-100.0 \%)$ in the discrimination between two groups at the time. 


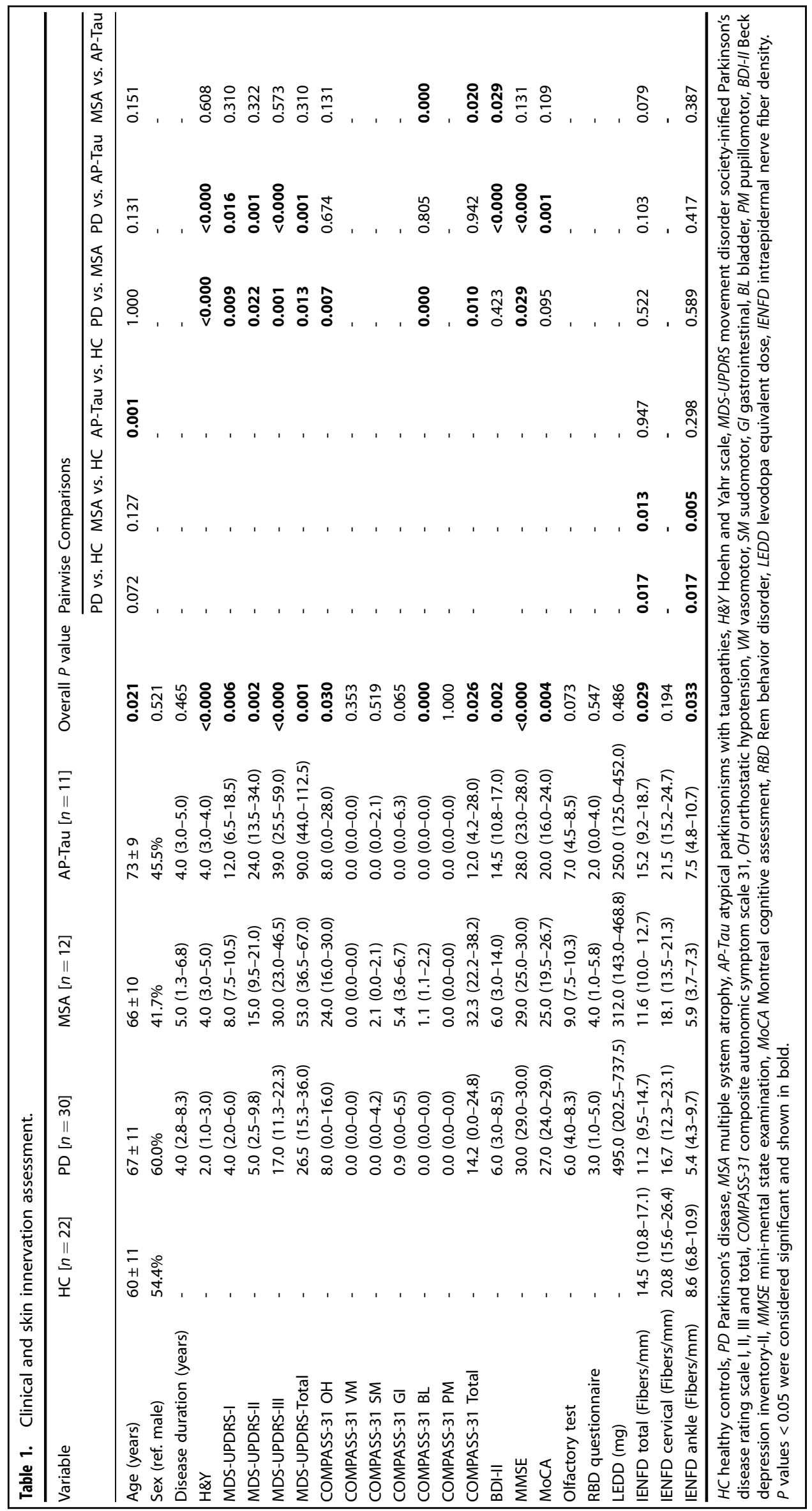




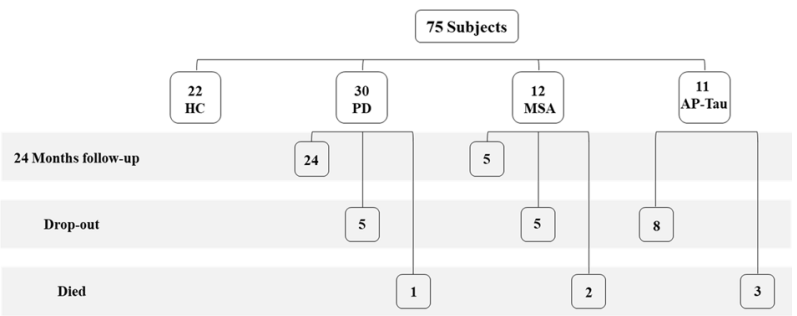

Fig. 1 Flowchart of the study population. Study groups are shown longitudinally in time.

\section{DISCUSSION}

We demonstrated that aSyn oligomers, detected in skin biopsy by PLA, are significantly more expressed in PD and MSA patients than in $\mathrm{HC}$ and AP-Tau and they yield a high diagnostic performance for synucleinopathies. Furthermore, we documented that significant denervation in both cervical and ankle sites occurs after two years of follow-up in PD patients and that low IENFD at TO is associated with progression of disease at cognitive and motor scales.

The observation of aSyn oligomers in skin peripheral nerves is in accordance with previous studies in the postmortem brain of

$\mathbf{a}$
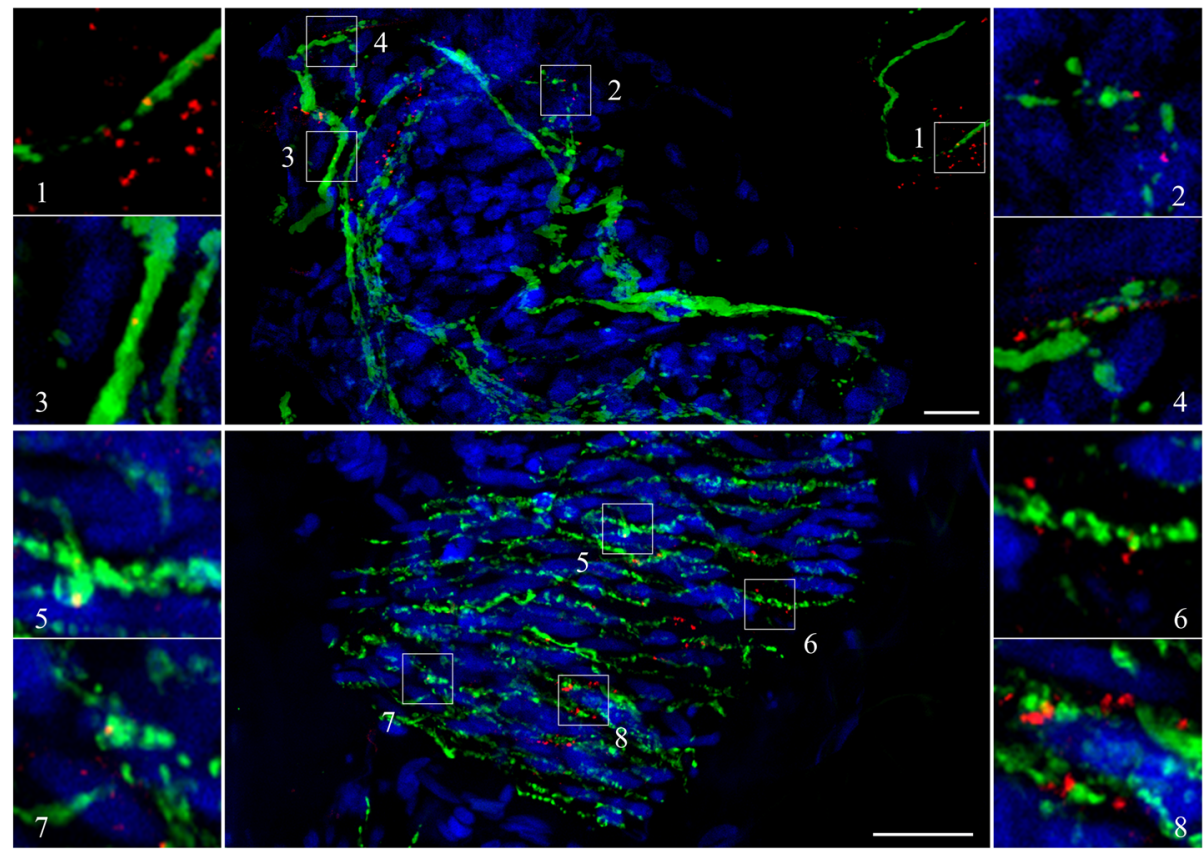

Co-localization
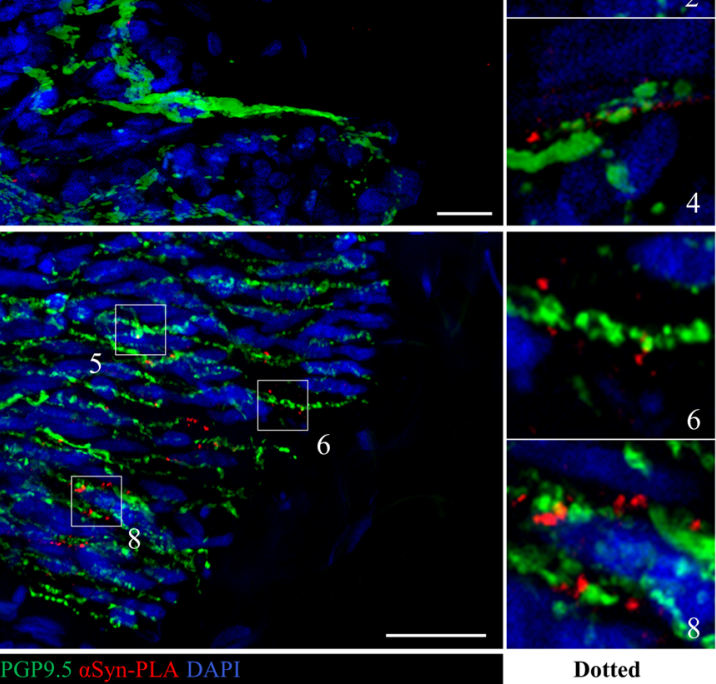

b
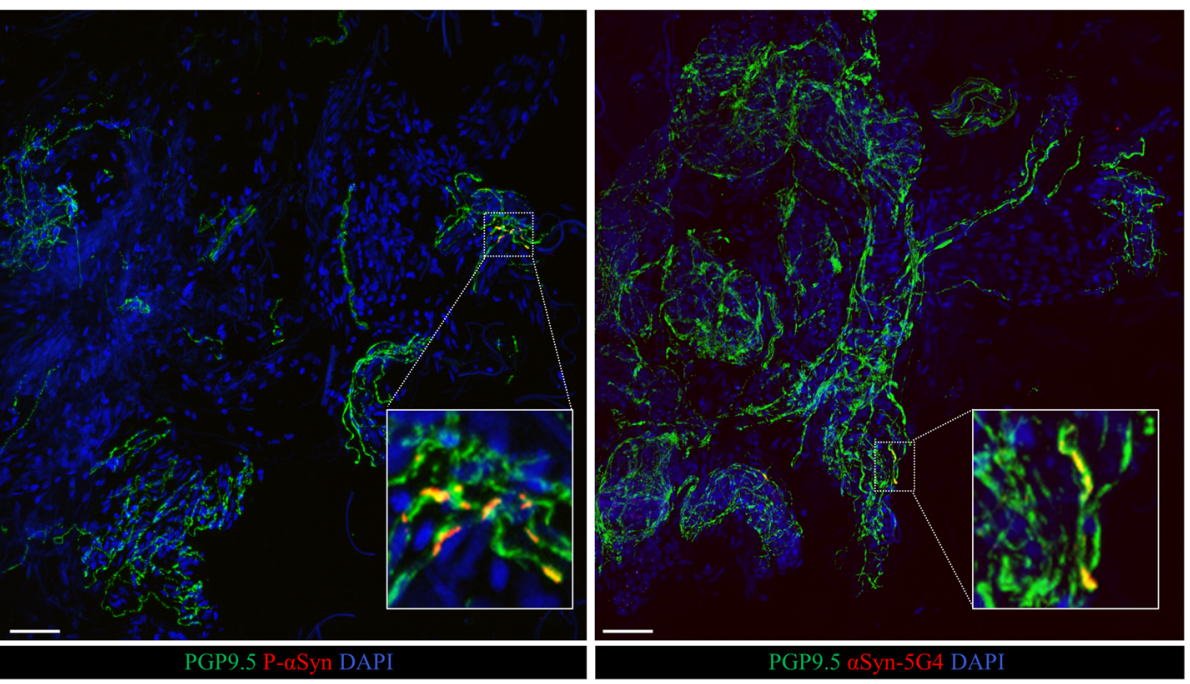

Fig. 2 aSyn PLA, P-aSyn and aSyn-5G4 signals in skin. a Confocal images of a sweat gland (top) and muscle arrector pili (bottom) in skin biopsy at the cervical site of a PD patient: in green the panaxonal marker protein gene product 9.5 (PGP9.5), in red $\alpha$ Syn oligomers detected by proximity ligation assay (PLA) technique. The Co-localization signal of $\alpha$ Syn-PLA and nerve fibers (magnification on the left) was defined by a yellow signal. The dotted signal (magnification on the right) was defined by an $\alpha$ Syn-PLA signal in the proximity of degenerating nerve fibers, characterized by swelling and fragmentations, or interposed between nerve fiber breakpoints. Scale bar $50 \mu \mathrm{mm}$. b Confocal images of sweat glands in cervical skin biopsy of a PD patient. In red, on the left phosphorylated $\alpha$ Syn, and on the right aggregated $\alpha$ Syn detected by 5G4 antibodies. Co-localization of P- $\alpha$ Syn/PGP9.5 and $\alpha$ Syn-5G4/PGP9.5 is shown in yellow. Scale bar $50 \mu$ m. 
$\mathbf{a}$

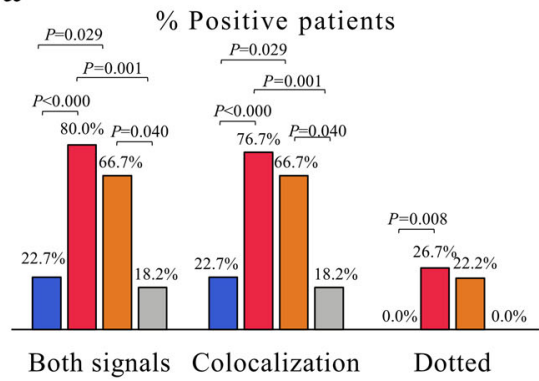

b

$\%$ Positive patients at cervical site

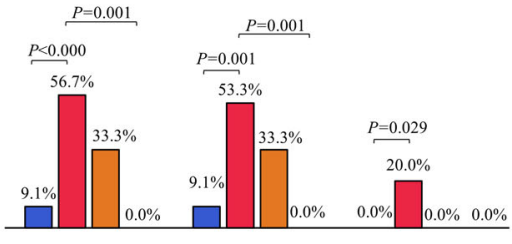

Both signals Colocalization Dotted

e

d

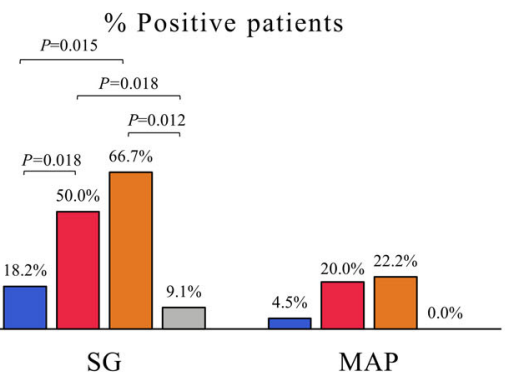

g

$\alpha$ Syn-PLA area
$\%$ Positive patients at cervical site

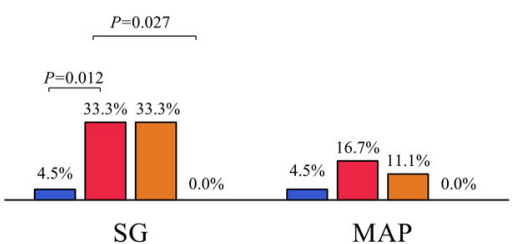

h

$\alpha$ Syn-PLA area at cervical site
$\%$ Positive patients at ankle site

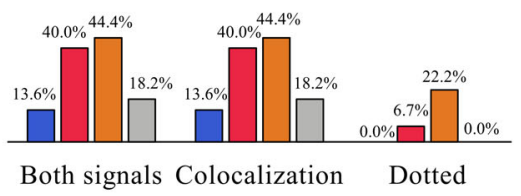

f

$\%$ Positive patients at ankle site

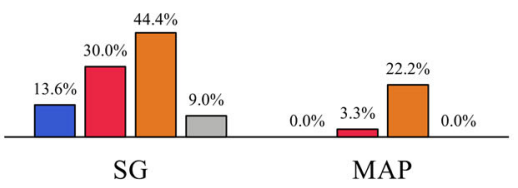

i $\alpha$ Syn-PLA area at ankle site
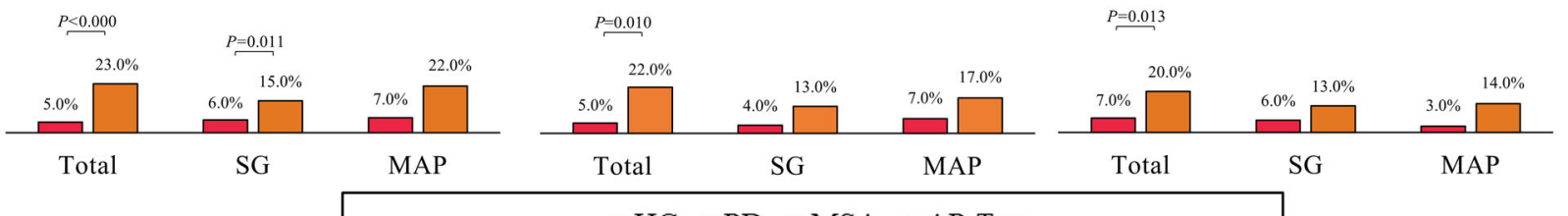

$\square \mathrm{HC} \square \mathrm{PD} \square \mathrm{MSA} \square \mathrm{AP}-\mathrm{Tau}$

Fig. 3 aSyn-PLA signal is significantly more expressed in PD and MSA. a-c $\alpha$ Syn-PLA signal is higher in PD and MSA. d-f The signal is observed mainly in the autonomic fibers surrounding SG and to a lesser extent MAP independently from the anatomical site. $\mathbf{g}-\mathbf{i}$ The area of intra-nervous oligomeric $\alpha$ Syn is significantly higher in MSA patients than PD, at both sites.

\begin{tabular}{|c|c|c|c|c|}
\hline PD vs. HC & & $P$ value & Odd ratio & $95 \% \mathrm{Cl}$ \\
\hline \multirow[t]{2}{*}{ Both localizations } & All structures & 0.000 & 13.60 & $3.56-51.92$ \\
\hline & SG & 0.023 & 4.500 & $1.23-16.49$ \\
\hline \multirow[t]{2}{*}{ Cervical } & All structures & 0.002 & 13.08 & $2.58-66.28$ \\
\hline & SG & 0.032 & 10.5 & $1.23-89.68$ \\
\hline PD vs. AP-Tau & & $P$ value & Odd ratio & $95 \% \mathrm{Cl}$ \\
\hline \multirow[t]{2}{*}{ Both localizations } & All structures & 0.001 & 18.00 & $3.05-106.12$ \\
\hline & SG & 0.038 & 10.000 & $1.134-88.167$ \\
\hline MSA vs. HC & & $P$ value & Odd ratio & $95 \% \mathrm{Cl}$ \\
\hline \multirow[t]{2}{*}{ Both localizations } & All structures & 0.028 & 6.800 & $1.223-37.497$ \\
\hline & SG & 0.014 & 9.000 & $1.550-52.266$ \\
\hline MSA vs. AP-Tau & & $P$ value & Odd ratio & $95 \% \mathrm{Cl}$ \\
\hline \multirow[t]{2}{*}{ Both localizations } & All structures & 0.037 & 9.000 & $1.140-71.038$ \\
\hline & SG & 0.018 & 20.000 & $1.676-238.630$ \\
\hline
\end{tabular}

$P$ values $<0.05$ are considered significant and shown in bold.
PD patients demonstrating aSyn-PLA signal in neuroanatomical regions usually mildly affected by pathology and in the absence of Lewy bodies ${ }^{25}$. These results underline how the aSyn-PLA technique specifically detects oligomers, which are supposedly involved at early phases of pathology, preceding the formation of insoluble aggregates in the brain. Notably, also in postmortem brains of MSA, where the pathological hallmark is represented by glial cytoplasmatic inclusions of aSyn, PLA methodology detected a widespread distribution of aSyn oligomers in neurons, as well as in oligodendrocytes ${ }^{27}$. Thus, albeit different molecular mechanisms of disease preferentially affecting glial cells are supposed to be implied in MSA versus PD, our results of overexpression of oligomeric aSyn in peripheral nerves in both groups are not surprising. Similarly, to what has been demonstrated in brains ${ }^{27}$, we observed larger colocalization areas of PLA signal within nerves in MSA than in $P D$, suggesting a larger amount of oligomeric aSyn in MSA, possibly reflecting different aSyn strains. The finding of the positive aSyn-PLA signal also in the HC group, even if in a nonsignificant low rate $(10-20 \%$ depending on considering one or two sites), deserves consideration. We observed it mainly at the ankle, inside nerves, and never in association with fragmented, degenerating axons (dotted signal), differently from synucleinopathies. The presence of incidental 
a

$\%$ Positive patients

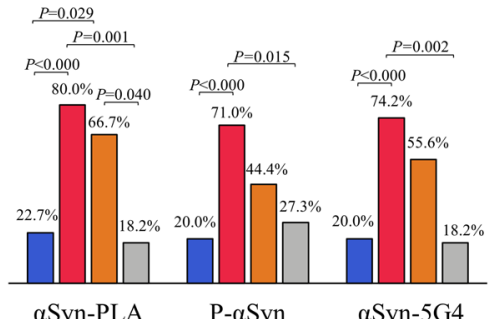

d

$\%$ Positive patients

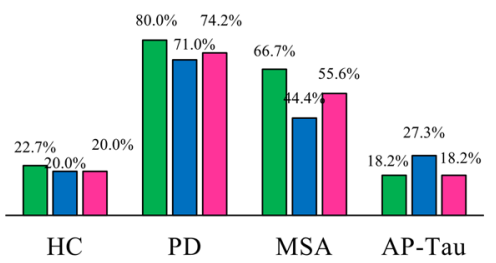

g

PD vs. HC Both localizations

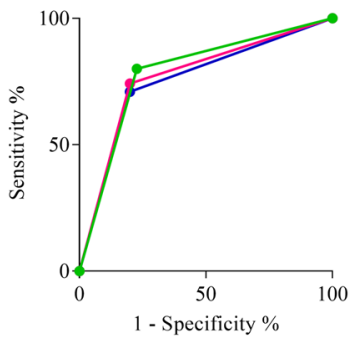

j

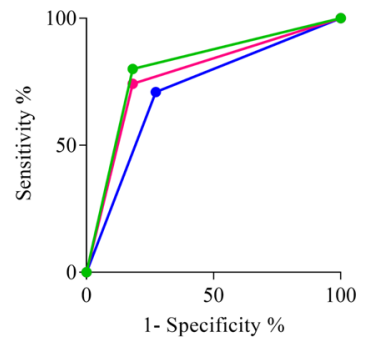

m

MSA vs. HC Both localizations

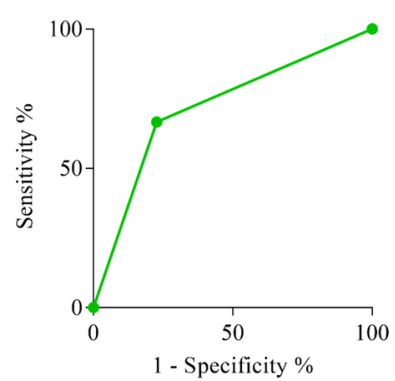

b

$\%$ Positive patients at cervical site

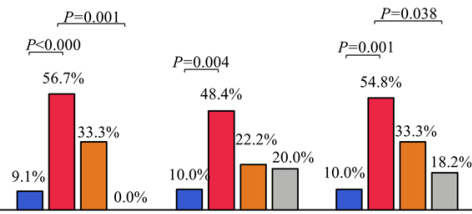

aSyn-PLA

P- $\alpha$ Syn

aSyn-5G4

$\square \mathrm{HC} \square \mathrm{PD} \square \mathrm{MSA} \square \mathrm{AP}-\mathrm{Tau}$

e

$\%$ Positive patients at cervical site
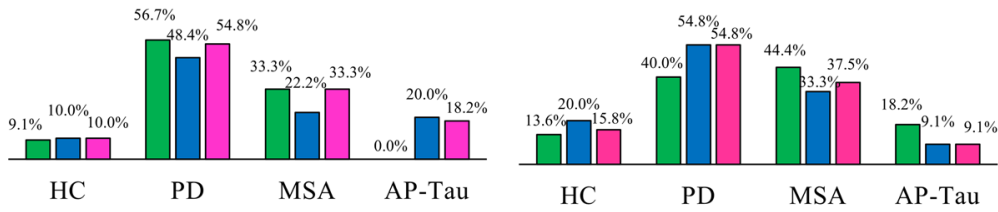

$\square$ aSyn-PLA $\quad \square$ P- $\alpha$ Syn $\quad \square \alpha$ Syn-5G4

h

PD vs. HC Cervical

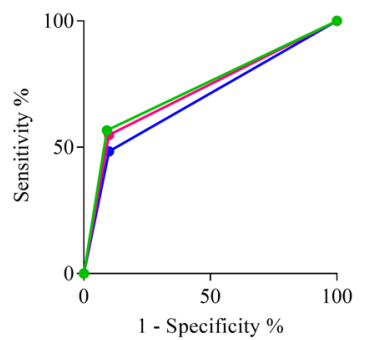

k

PD $v$. AP-Tau Cervical

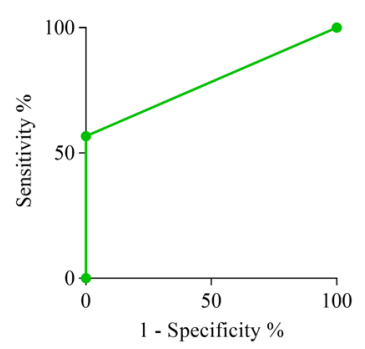

C $\%$ Positive patients at ankle site

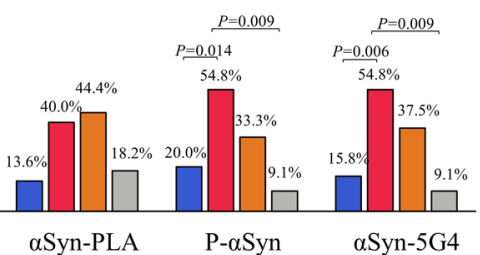

$\mathbf{f}$ $\%$ Positive patients at ankle site i

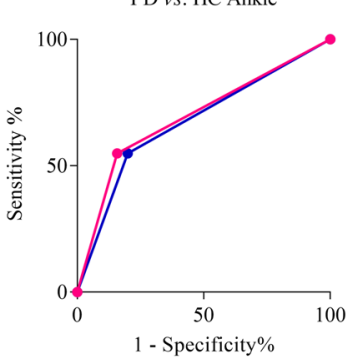

I

PD vs. AP-Tau Ankle

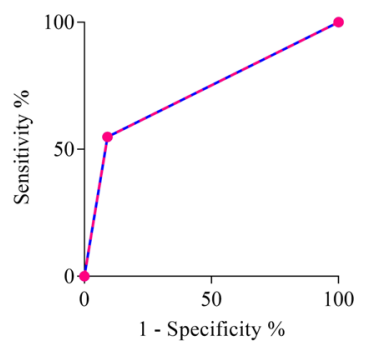

\begin{tabular}{|c|c|c|c|c|c|}
\hline & \\
\hline & & & AUC & Sens. $\%$ & Spec. $\%$ \\
\hline \multirow{3}{*}{ PD vs. HC } & \multirow{3}{*}{$\begin{array}{c}\text { Both } \\
\text { localizations }\end{array}$} & $\alpha$ Syn-PLA & 0.786 & 80.0 & 77.3 \\
\hline & & $\mathrm{P}-\alpha$ Syn & 0.755 & 70.9 & 80.0 \\
\hline & & $\alpha$ Syn-5G4 & 0.771 & 74.2 & 80.0 \\
\hline \multirow{3}{*}{ PD vs. HC } & \multirow{3}{*}{ Cervical } & $\alpha$ Syn-PLA & 0.738 & 56.7 & 90.9 \\
\hline & & P- $\alpha$ Syn & 0.691 & 48.4 & 90.0 \\
\hline & & $\alpha$ Syn-5G4 & 0.724 & 54.8 & 90.0 \\
\hline \multirow{2}{*}{ PD vs. HC } & \multirow{2}{*}{ Ankle } & $\mathrm{P}-\alpha$ Syn & 0.674 & 54.8 & 80.0 \\
\hline & & $\alpha$ Syn-5G4 & 0.695 & 54.8 & 84.2 \\
\hline \multirow{3}{*}{ PD vs AP-Tau } & \multirow{3}{*}{$\begin{array}{c}\text { Both } \\
\text { localizations }\end{array}$} & $\alpha$ Syn-PLA & 0.809 & 80.0 & 81.2 \\
\hline & & P- $\alpha$ Syn & 0.718 & 70.9 & 72.7 \\
\hline & & $\alpha$ Syn-5G4 & 0.780 & 74.2 & 81.8 \\
\hline PD vs AP-Tau & Cervical & $\alpha$ Syn-PLA & 0.783 & 56.6 & 100.0 \\
\hline \multirow{2}{*}{ PD vs AP-Tau } & \multirow{2}{*}{ Ankle } & P- $\alpha$ Syn & 0.728 & 54.8 & 90.9 \\
\hline & & $\alpha$ Syn-5G4 & 0.728 & 54.8 & 90.9 \\
\hline MSA vs HC & $\begin{array}{c}\text { Both } \\
\text { localizations }\end{array}$ & $\alpha$ Syn-PLA & 0.719 & 66.7 & 77.3 \\
\hline
\end{tabular}

Fig. 4 Comparative analysis and diagnostic performance of aSyn-PLA, P-aSyn, and 5G4. a $\alpha$ Syn-PLA presented the highest sensitivity for PD and MSA at both locations and allowed the differentiation from HC and AP-Tau. $\mathbf{b}$ At cervical site $\alpha$ Syn-PLA and $\alpha$ Syn-5G4 showed higher sensitivity than P- $\alpha$ Syn for PD. c In the ankle, P- $\alpha$ Syn and $\alpha$ Syn-5G4 were more sensitive for PD then $\alpha$ Syn -PLA. d-f Within groups no differences were observed between markers, but $\alpha$ Syn-PLA showed a higher sensitivity for MSA than P- $\alpha$ Syn and $\alpha$ Syn-5G4. g-i ROC curve analysis of PD vs. HC, j-I PD vs. AP-Tau, m MSA vs. HC. For each marker and location, AUC, sensitivity, and specificity are reported. 


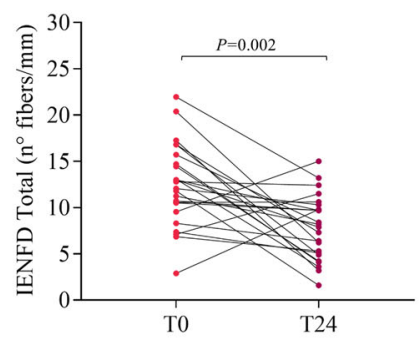

d $\%$ Positive patients

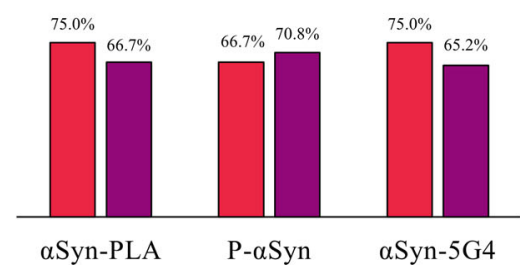

g

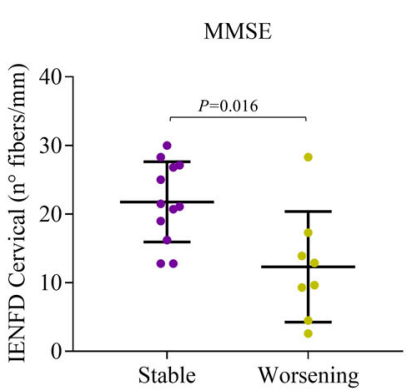

j

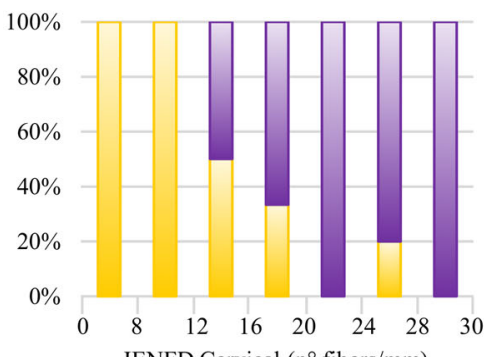

b

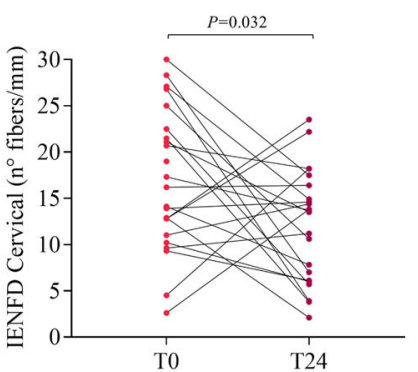

e

$\%$ Positive patients at cervical site

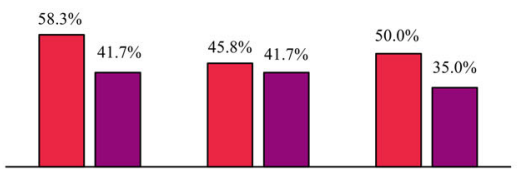

$\alpha$ Syn-PLA $\quad$ P- $\alpha$ Syn $\quad \alpha$ Syn-5G4

$\square \mathrm{T} 0 \quad \mathrm{~T} 24$

h

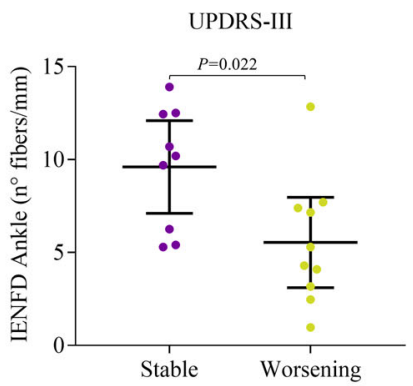

c

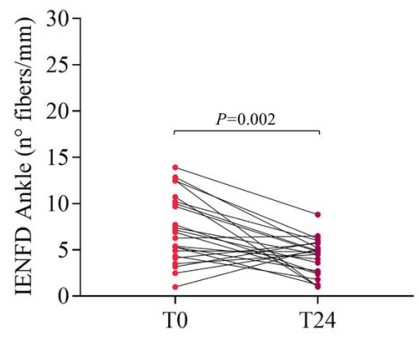

f

$\%$ Positive patients at ankle site

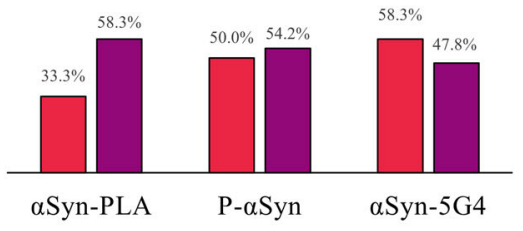

i

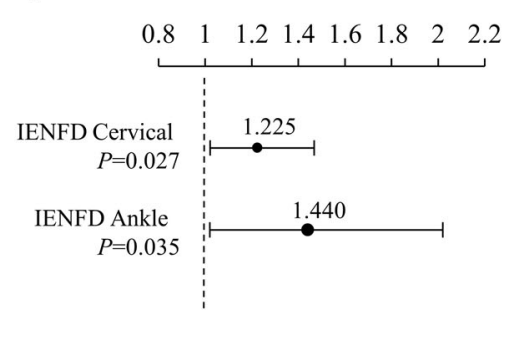

口Stable MMSE

$\square$ Worsening MMSE k

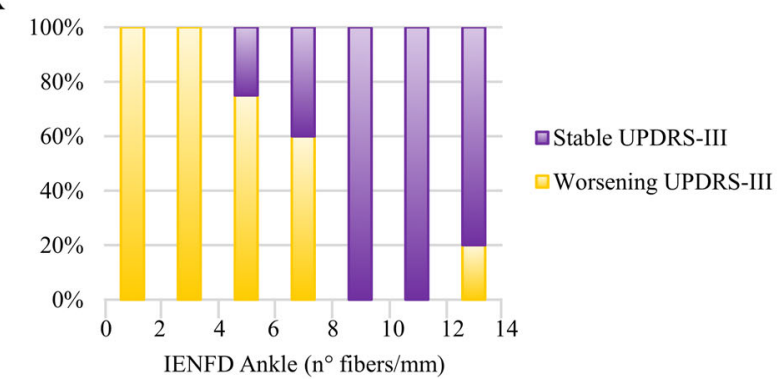

Fig. 5 PD patients present a progression of denervation not of pathological aSyn at T24 and IENFD predicts cognitive and motor decline. a After 24 months PD patients showed a significant progression of intraepidermal denervation, $\mathbf{b}, \mathbf{c}$ at both anatomical sites. $\mathbf{d}-\mathbf{f}$ No significant differences were observed in the percentage of positivity for $\alpha$ Syn-PLA, P- $\alpha$ Syn, and $\alpha$ Syn-5G4 but a slight reduction for all markers at the cervical site and an increase of $\alpha$ Syn-PLA in the ankle are noted. g Patients with a worsening of MMSE scale at T24 showed a lower cervical IENFD at T0; $\mathbf{h}$ while a lower ankle IENFD was present at T0 in patients with a progressive motor impairment at MDS-UPDRS-III scale. i Univariate logistic regression analysis showed an association between cervical IENFD and MMSE, and between ankle IENFD and MDS-UPDRSIII; ODD ratio and $P$ value are reported. $\mathbf{j}, \mathbf{k}$ Percentage of patients with cognitive and motor progression at T24 are represented according to respectively cervical and ankle IENFD at T0.

aSyn pathology has been detected in brain autopsies of $10-20 \%$ of subjects $>70$ years, without neurological diseases ${ }^{30}$. Roberts et al. described a diffuse kind of aSyn-PLA signal in HC too, mainly located in the neuropil around neurons and less frequently in the white matter of brain areas usually affected by PD pathology ${ }^{25}$. The neuropil is mainly composed of unmyelinated axons, dendrites, and synapsis, which are very similar to the composition of small fiber nerves, either unmyelinated $C$ fiber or small myelinated $A \delta$, present in the skin. Indeed, aggregates of aSyn, are preferentially formed in projection neurons with very long, thin unmyelinated axons, which are more susceptible to axonal transport deficit, energetic/metabolic stress, mitochondrial failure, and lack the trophic support of glial cells $s^{31}$. Finally, Mazzetti et al. detected a nonsignificant quote of aSyn-PLA signal in skin 
a

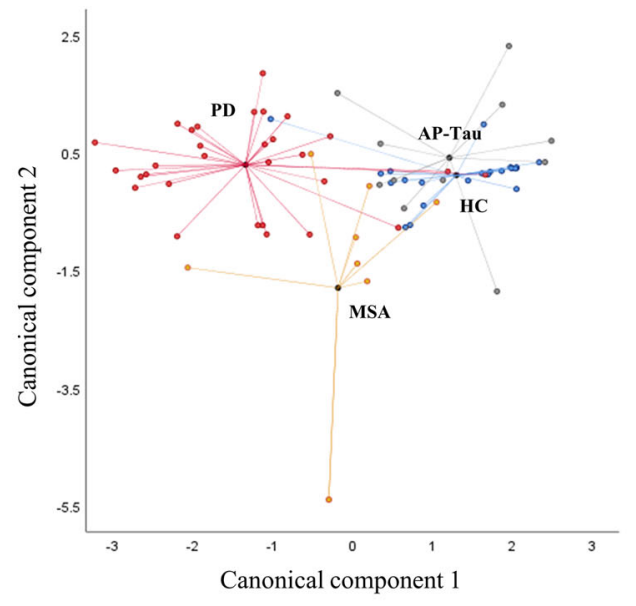

b

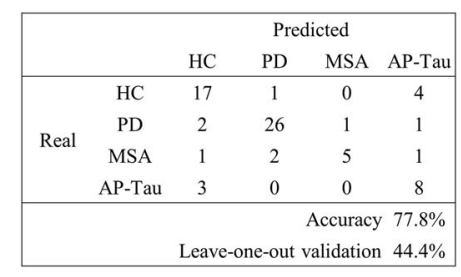

c

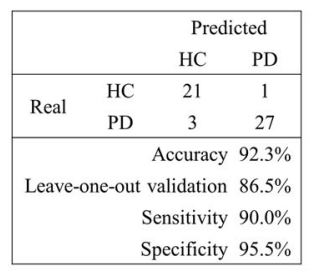

d

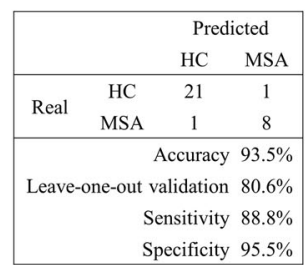

e

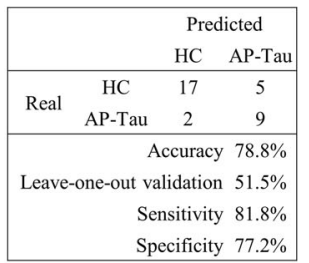

f

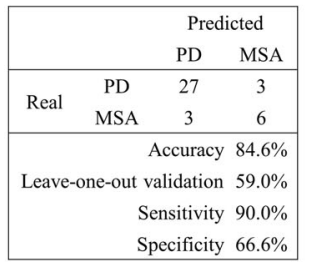

g

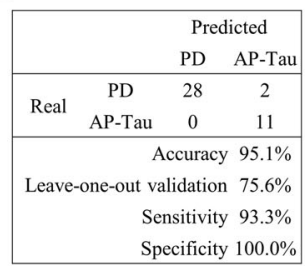

h

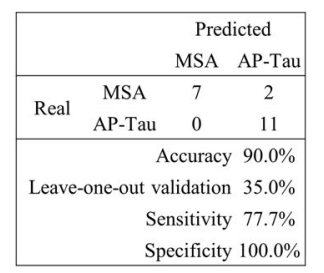

Fig. 6 Skin biopsy-derived compound marker allows stratification of patients according to the diagnosis. a Canonical plot showing patients according to the diagnosis. The model was built considering the presence/absence of $\alpha S y n-P L A, P-\alpha S y n, \alpha S y n-5 G 4$, and intraepidermal nerve fiber density (IENFD) at both anatomical sites. The axes of the plot (canonical components 1 and 2) were calculated from weighted linear combinations of variables to maximize the separation between the four groups. Each subject is represented by a point. b-h Confusion matrix reporting real and predicted diagnosis, accuracy, internal validation by the leave-one-out algorithm, sensitivity, and specificity is reported for all groups and pairwise comparisons.

autonomic nerves of $\mathrm{HC}^{29}$. Thus, aSyn oligomers in $\mathrm{HC}$ may represent aberrant oligomerization of aSyn at the synapses or axonal transport of pathological species as a very early subclinical event that may become more relevant with aging and/or external toxic events in a susceptible population. However, our study cohort is mainly composed of established PD with long disease duration, and larger studies including prodromal phases of disease using aSyn-PLA technology in skin biopsies are warranted.

The anatomical site providing the best differentiation between $P D$ vs. $H C$ and AP-Tau is the cervical one while both anatomical sites are required to differentiate $\mathrm{HC}$ and AP-Tau from MSA, in which a higher presence of oligomeric aSyn at the ankle rather than at the cervical site is shown, differently from PD. This is in line with previous observations of a distal-to-proximal gradient of aSyn aggregates in $\mathrm{MSA}^{32}$, possibly consequent of a different spreading of aSyn oligomers, or because different aSyn strains have been postulated in MSA versus PD $^{33}$.

The dermal autonomic structures presenting most of the PLA signal are SG in line with Mazzetti et al. ${ }^{29}$ followed by MAP. This result is in accordance with previous work showing P-aSyn mainly in SG of PD patients ${ }^{34}$. We found a similar major distribution of oligomeric aSyn in SG also in MSA, while the distribution of P-aSyn in autonomic or somatosensory nerve fibers in this group is more debated in the literature and some authors have shown a predominance of P-aSyn in somatosensory nerve fibers instead of autonomic ones ${ }^{12}$. However, while aSyn oligomers are small aggregates preceding the formation of fibrils in the early stages of pathology, P-aSyn accumulates in Lewy bodies, and yet it is still debated whether phosphorylation at Serine 129 enhances or suppresses aSyn aggregation and toxicity ${ }^{35}$, as this could be a later event in disease progression. Thus, aSyn-PLA and P-aSyn are probably markers of different stages of the disease. A recent study focused on aSyn content in MAP found that patients with Lewy- body neurogenic orthostatic hypotension displayed higher content of aSyn in sympathetic noradrenergic nerves than $\mathrm{MSA}^{36}$. Thus, based on the selection of different protein targets and different anatomical sites, skin biopsy analysis can distinguish between PD and MSA.

The comparative analysis of the diagnostic performance of aSyn-PLA vs. P-aSyn and $5 G 4$ in discriminating PD from $\mathrm{HC}$ and AP-Tau has shown that aSyn-PLA displays the best accuracy when considering both locations and the cervical site alone. This result can also be explained by the fact that by detecting oligomers, the early species in the aggregation pathway, PLA can bear more sensitivity to early phases of disease than P-aSyn. However, P-aSyn and 5G4, possible markers of later phases of the disease, performed better when considering the ankle site alone. Thus, the choice of pathological aSyn marker and the site of skin biopsy influences the diagnostic performance importantly and can help to understand the temporal dynamics of a aSyn seeding and spreading in the peripheral nervous system during the course of the disease. Of note, aSyn-PLA was the only technique yielding discrimination between MSA and $\mathrm{HC}$. These are remarkable results for aSyn-PLA considering that a very recent study has shown that P-aSyn detection in the skin has a higher diagnostic accuracy for synucleinopathies than real-time quaking-induced conversion (RTQuIC) in the skin, a sensible and accurate technology to measure prion-like self-aggregation of pathological $\mathrm{aSyn}^{37}$. However, our results may be partially influenced by methodological issues, since aSyn-PLA signal was analyzed by confocal microscopy while PaSyn and 5G4 were analyzed by fluorescence microscopy so that a better resolution was guaranteed in the first condition. It should be taken into account that in both cases the interpretation of immunofluorescence findings is a complex process and requires a trained pathologist. 
We further confirmed the presence of a small fiber neuropathy in PD and MSA, as observed in previous studies with skin biopsy ${ }^{14,22,38}$. The neuropathy is not related to levodopa therapy or vitamin B12 deficiency, and it is very likely secondary to the neurodegeneration process ${ }^{39,40}$. More importantly, we showed that denervation progresses in a significant way with disease duration, even in a relatively short period of time ( 2 years), while no significant changes in clinical scales or in pathological aSyn amounts were detectable in this study. Furthermore, we demonstrated that a lower ankle IENFD at TO was associated with a progression of motor impairment at the MDS-UPDRS-III scale and that a lower cervical IENFD was associated with a progression of cognitive decline at MMSE, independently from age and LEDD. These findings are in line with brain pathology studies showing that clinical symptoms in PD align with neuronal degeneration rather than with the aSyn aggregation burden ${ }^{41}$. Thus, the relevance of IENFD as a progression marker in PD is worthy of further investigations in larger studies, because if the clinical significance of IENFD changes is confirmed, skin denervation could be considered as a proxy measure of neurodegenerative events occurring in the brain and possibly an ancillary biomarker in clinical trials. Moreover, the measure of IENFD by skin biopsy bears the advantage of being easily and rapidly performed at low cost, as well as being extensively studied and standardized in the last twenty years for the diagnosis of small fiber neuropathy ${ }^{42,43}$. Nevertheless, an important limitation of the current study is that the most severely affected patients of the cohort were lost at follow-up, potentially limiting the ability to evaluate more aggressive forms with higher progression rates.

Of note, a compound marker based on all three markers for pathological aSyn and IENFD was able to stratify patients with high accuracy according to the diagnosis and to discriminate between PD and MSA (84.6\%), which is a major challenge in clinical practice. In conclusion, skin biopsy represents a minimallyinvasive, easily-accessible, and repeatable source of biomarkers for PD in vivo: multiple target epitopes of proteins involved in the pathogenesis of the disease are detectable and it allows the quantification of small fiber neuropathy through IENFD, which represents a promising prognostic marker. A thorough evaluation of multiple markers bearing complementary information and of small fiber pathology by skin biopsy is advisable not only for the diagnosis of PD in routine clinical practice but also for the future development of more adequate biomarkers as surrogate endpoints in pharmacological clinical trials for PD.

\section{METHODS}

\section{Patients' recruitments}

Thirty patients with idiopathic PD, 22 age and sex-matched HC, and 23 AP were prospectively recruited from the movement disorders outpatient clinic at the Neurocenter of Southern Switzerland in Lugano, from July 2015 to January 2021 as part of the NSIPD001 study ${ }^{14}$. Inclusion criteria for PD were a clinical diagnosis according to the UK Brain Bank diagnostic criteria ${ }^{44}$, disease duration of at least 2 years, no family history, and no major cognitive impairment or major autonomic dysfunction symptoms in the history. $\mathrm{HC}$ subjects were recruited among patients' partners and hospital staff without any known pathology. AP group included 12 patients with probable MSA and 11 patients with possible AP-Tau, among which eight with probable progressive supranuclear palsy (PSP) and three with possible corticobasal degeneration (CBD). Inclusion criteria for AP were based on published diagnostic criteria for $\mathrm{MSA}^{45}, \mathrm{PSP}^{46}$, and $\mathrm{CBD}^{47}$. Exclusion criteria were co-morbidities causing peripheral polyneuropathies (diabetes, renal failure, thyroid pathology, vitamin B12 deficiency, HIV and HCV infection, Lyme disease, syphilis, acute or chronic inflammatory diseases) and tumors. After 2 years (T24) PD and AP patients underwent a follow-up clinical evaluation and a second skin biopsy (Fig.1).

The Cantonal Ethics Committee approved the study protocol, and all enrolled subjects gave written informed consent to the study.

\section{Clinical assessment}

Disease severity was determined by the Hoehn and Yahr Scale $(H \& Y)^{48}$ and the Movement Disorder Society Unified Parkinson's Disease Rating Scale $\left(\right.$ MDS-UPDRS) ${ }^{49}$. Cognitive impairment was assessed with the Mini-Mental State Examination (MMSE) $)^{50}$ and the Montreal Cognitive Assessment $(\mathrm{MoCA})^{51}$. Symptoms of autonomic dysfunction were rated with the Composite Autonomic Symptom Score 31 (COMPASS-31) ${ }^{52}$. Mood disorders were screened with the Beck depression inventory-II (BDI-II) ${ }^{53}$, while REM sleep behavior disorder (RBD) with the RBD screening questionnaire; ${ }^{54}$ olfactory function was tested with the Sniffin' Sticks Smell test (Burghart Messtechnik $\mathrm{GmbH}$, Wedel, Germany). The levodopa equivalent daily dose (LEDD) was calculated for all PD and AP patients ${ }^{55}$.

\section{Skin biopsy}

A 3-mm punch skin biopsy was performed on the clinically more affected side, as previously described ${ }^{14,56}$. Each subject underwent skin collection at two anatomical sites, namely at the C8 dermatomal level (cervical) and at the distal leg, about $10 \mathrm{~cm}$ above the lateral malleolus (ankle). Skin samples were fixed overnight at $4^{\circ} \mathrm{C}$ in Paraformaldehyde-lysine-periodate (PLP) $2 \%$ fixative. The day after, skin samples were frozen and cut with a cryotome to obtain $50-\mu \mathrm{m}$-thin tissue sections for free-floating immunofluorescence analysis ${ }^{14,42}$. All the immunofluorescence staining were manually performed by an operator blind to the clinical diagnosis

\section{Intraepidermal nerve fiber density}

Three non-consecutive $50-\mu \mathrm{m}$-thin tissue sections per localization per patient were stained with the antibody against protein gene product 9.5 (PGP9.5; Abcam, Cambridge UK, 1:1000) ${ }^{56}$. Cell nuclei were counterstained with 4',6-diamidino-2-phenylindole (DAPI, Sigma Aldrich, Saint Louis USA, 1:5000). According to published standard protocols ${ }^{42,57}$, PGP9.5 positive nerve fibers, crossing the dermal-epidermal junction, were counted to determine the linear IENFD. The length of the section was measured and IENFD was obtained by dividing the number of fibers by the length of the section and expressed as "number of fibers $/ \mathrm{mm}$ ". IENFD was determined at both ankle and cervical sites and total IENFD was calculated as the mean of nerve densities at both localizations.

\section{Direct proximity ligation assay (PLA)}

Direct PLA was performed with the Duolink PLA kit (Duolink Kit Sigma Aldrich, Saint Louis USA). Following the manufacturer's instructions, the oligonucleotides probes MINUS and PLUS were separately conjugated to the mouse monoclonal aSyn antibody with blocking activity (anti-aSyn $211,1 \mathrm{mg} / \mathrm{mL}$, Abcam, Cambridge, UK) ${ }^{25}$. Three non-consecutive $50-\mu \mathrm{m}$ thin tissue sections per localization per patient were washed in TBS and then incubated for $2 \mathrm{~h}$ at room temperature in a freshly made blocking solution (4\% NGS, $1 \%$ Triton in TBS). Sections were incubated with aSynPLUS and aSyn-MINUS probes (1:100 in PLA diluent) and rabbit anti-PGP9.5 antibody (Abcam, Cambridge, UK, 1:1000) for $1 \mathrm{~h}$ at $37^{\circ} \mathrm{C}$ and then overnight at room temperature.

The day after, the amplification reaction was performed by serial incubation with (1) ligase enzyme diluted 1:40 in ligation buffer (Duolink kit, Sigma Aldrich, Saint Louis USA) for $1 \mathrm{~h}$ at $37^{\circ} \mathrm{C}$; (2) polymerase enzyme diluted 1:80 in the Amplification buffer (Duolink kit, Sigma Aldrich, Saint Louis USA) and secondary antibodies AlexaFluor488 goat anti-rabbit (ThermoFisher Scientific, Waltham USA, $1: 700$ ) for $2 \mathrm{~h}$ at $37^{\circ} \mathrm{C}$. Finally, cell nuclei were counterstained with DAPI (1:5000 in PBS for $4 \mathrm{~min}$ ) and section slides were mounted with Vectashield (Vector Laboratories, Burlingame, USA).

The specificity of the assay for aSyn-PLA was first assessed in paraffined brain sections from PD and HC (Supp. Fig. 1). Serial negative controls without oligonucleotide probes and amplification reaction enzymes were also conducted (Supp. Fig. 1).

\section{Confocal microscope analysis of aSyn-PLA}

To increase the specificity of the analysis we considered only aSyn-PLA signal within the nerves which colocalized with the axonal marker PGP9.5 (colocalization signal) or which was located in proximity to degenerated nerve fibers, defined by axonal swellings and fragmentations or interposed between nerve fiber breakpoints (dotted signal) (Fig. 2a).

Skin sections were first rated under an inverted fluorescence microscope (Nikon Eclipse Ti-E, Tokyo, Japan). For each section, both somatosensory and autonomic innervation were screened: intraepidermal nerve fibers, 
dermal nerve bundles, and autonomic structures (sweat glands [SG], muscle arrector pili [MAP], and vessels). All SG and MAP were analyzed by a Nikon confocal microscope using the NIS Elements 4.11.01 imaging software. Serial pictures, every $2 \mu \mathrm{m}$ on the Z-axis, were taken using a $20 \mathrm{X}$ magnification. To avoid missing small amounts of signal within dermal nerve bundles, we also analyzed at least three nerve bundles that were randomly chosen at each anatomical site by confocal microscope. Every single stack of all the obtained pictures was analyzed with ImageJ software by two independent examiners, blinded to the clinical diagnosis. For each image, we reported the presence/absence of signal. Structures were defined as "positive" if showing aSyn-PLA. Anatomical sites were defined as "positive" when at least one positive structure was present. The subjects were defined as "positive" when they showed at least one positive site. Negative subjects did not present PLA signals at any site. To assess the anatomical distribution of PLA signal in each group, we also considered ankle and cervical sites separately.

\section{Quantification of aSyn-PLA}

For each patient, the percentage of positive SG and MAP structures was calculated. For each structure, the number of colocalization points was counted and, if one subject showed signal in multiple structures and sites, the sum of the points was considered. Finally, we calculated the area occupied by aSyn-PLA signal normalized for the area of innervation. First, the stacks on the Z-axis were merged, then the area of the structure (DAPI), the area of innervation (PGP9.5), and the area of aSyn-PLA within nerves were measured with the "freehand selection" tool. The area of innervation was normalized for the area of the structure and then the percentage of the innervation area occupied by aSyn-PLA signal was measured.

\section{Phosphorylated alpha-synuclein and 5G4 immunofluorescence}

Three non-consecutive $50-\mu \mathrm{m}$-thin tissue sections per localization per patient were incubated overnight with antibodies against PGP9.5 (1.1000, Abcam, Cambridge, UK), P-aSyn at serine 129 (1.1000, Wako Chemicals, Neuss, Germany) or 5G4 (1.400, Analytik Jena Life Science, Jena, Germany $)^{56}$. The day after, sections were incubated with secondary antibody AlexaFluor488 and AlexaFluor594 (ThermoFisher Scientific, Waltham USA, 1:700), and counterstained with DAPI. All sections were analyzed at a fluorescence microscope by two independent raters blinded to the diagnosis. Colocalization of P-aSyn/5G4 and PGP9.5 was considered as positive signal as previously described ${ }^{14}$.

\section{Statistical analysis}

Statistical analyses were performed with IBM SPSS Statistics 26.0 (IBM Corp. Released 2019. IBM SPSS Statistics for Windows, Version 26.0. Armonk, NY: IBM Corp). Variable distribution was assessed by the Kolmogorov-Smirnov test. One-way ANOVA test with post hoc Bonferroni's test for multiple comparisons was used for normally distributed variables expressed as mean \pm standard deviation. Kruskal-Wallis' test was applied to nonnormally distributed variables expressed as medians and interquartile range. $X^{2}$ or Fisher's exact tests were used for categorical variables expressed as a percentage (\%). Odds ratios (OR) were assessed by univariate logistic regression analysis, while the area under the curve (AUC) and the diagnostic performances of selected variables were obtained with receiver operating characteristics (ROC) curves analysis. For matched analysis (T0 vs. T24), non-normally distributed variables were analyzed by Wilcoxon pairs signed-rank test, and categorical variables by McNemar test. Associations between IEFND (cervical, ankle, and total) at TO and worsening of clinical characteristics (MoCA, MMSE, MDS-UPDRS-III) at T24 were tested using logistic regression models adjusted by age and LEDD, with OR and $95 \%$ confidence intervals calculation $(95 \% \mathrm{Cl})$.

The linear discriminant analysis model was used to classify patients. Canonical components 1 and 2 were calculated from weighted linear combinations of variables (aSyn-PLA, P-aSyn, aSyn-5G4, and IENFD) to maximize the separation between groups.

\section{Reporting summary}

Further information on research design is available in the Nature Research Reporting Summary linked to this article.

\section{DATA AVAILABILITY}

The raw data that support the findings of this article are available on reasonable request to the corresponding author.

Received: 3 August 2021; Accepted: 25 November 2021; Published online: 20 December 2021

\section{REFERENCES}

1. Spillantini, M. G. \& Goedert, M. The alpha-synucleinopathies: Parkinson's disease, dementia with Lewy bodies, and multiple system atrophy. Ann. N. Y Acad. Sci. 920, 16-27 (2000).

2. Krismer, F. \& Wenning, G. K. Multiple system atrophy: insights into a rare and debilitating movement disorder. Nat. Rev. Neurol. 13, 232-243 (2017).

3. Klingelhoefer, L. \& Reichmann, H. Parkinson's disease as a multisystem disorder. J. Neural Transm. 124, 709-713 (2017).

4. Kumaresan, M. \& Khan, S. Spectrum of non-motor symptoms in Parkinson's disease. Cureus 13, e13275 (2021).

5. Beach, T. G. et al. Multi-organ distribution of phosphorylated alpha-synuclein histopathology in subjects with Lewy body disorders. Acta Neuropathol. 119, 689-702 (2010).

6. Lee, J. M. et al. The search for a peripheral biopsy indicator of alpha-synuclein pathology for Parkinson disease. J. Neuropathol. Exp. Neurol. 76, 2-15 (2017).

7. Shannon, K. M. et al. Alpha-synuclein in colonic submucosa in early untreated Parkinson's disease. Mov. Disord. 27, 709-715 (2012).

8. Irwin, D. \& Saint-Hilaire, M. In vivo detection of underlying synucleinopathies: are we there yet? Neurology 96, 925-926 (2021).

9. Stokholm, M. G., Danielsen, E. H., Hamilton-Dutoit, S. J. \& Borghammer, P. Pathological alpha-synuclein in gastrointestinal tissues from prodromal Parkinson disease patients. Ann. Neurol. 79, 940-949 (2016).

10. Del Tredici, K., Hawkes, C. H., Ghebremedhin, E. \& Braak, H. Lewy pathology in the submandibular gland of individuals with incidental Lewy body disease and sporadic Parkinson's disease. Acta Neuropathol. 119, 703-713 (2010).

11. Donadio, V. et al. Skin nerve alpha-synuclein deposits: a biomarker for idiopathic Parkinson disease. Neurology 82, 1362-1369 (2014).

12. Doppler, K. et al. Distinctive distribution of phospho-alpha-synuclein in dermal nerves in multiple system atrophy. Mov. Disord. 30, 1688-1692 (2015).

13. Gibbons, C. H., Garcia, J., Wang, N., Shih, L. C. \& Freeman, R. The diagnostic discrimination of cutaneous alpha-synuclein deposition in Parkinson disease. Neurology 87, 505-512 (2016).

14. Melli, G. et al. Cervical skin denervation associates with alpha-synuclein aggregates in Parkinson disease. Ann. Clin. Transl. Neurol. 5, 1394-1407 (2018).

15. Iwanaga, K. et al. Lewy body-type degeneration in cardiac plexus in Parkinson's and incidental Lewy body diseases. Neurology 52, 1269-1271 (1999).

16. Kovacs, G. G. et al. Intracellular processing of disease-associated alpha-synuclein in the human brain suggests prion-like cell-to-cell spread. Neurobiol. Dis. 69, 76-92 (2014).

17. Kovacs, G. G. et al. An antibody with high reactivity for disease-associated alphasynuclein reveals extensive brain pathology. Acta Neuropathol. 124, 37-50 (2012).

18. Kumar, S. T. et al. How specific are the conformation-specific alpha-synuclein antibodies? Characterization and validation of 16 alpha-synuclein conformationspecific antibodies using well-characterized preparations of alpha-synuclein monomers, fibrils and oligomers with distinct structures and morphology. Neurobiol. Dis. 146, 105086 (2020).

19. Iranzo, A. et al. alpha-Synuclein aggregates in labial salivary glands of idiopathic rapid eye movement sleep behavior disorder. Sleep https://doi.org/10.1093/ sleep/zsy101 (2018).

20. Skorvanek, M. et al. alpha-Synuclein antibody $5 \mathrm{G} 4$ identifies manifest and prodromal Parkinson's disease in colonic mucosa. Mov. Disord. 33, 1366-1368 (2018).

21. Tsukita, K., Sakamaki-Tsukita, H., Tanaka, K., Suenaga, T. \& Takahashi, R. Value of in vivo alpha-synuclein deposits in Parkinson's disease: a systematic review and meta-analysis. Mov. Disord. 34, 1452-1463 (2019).

22. Nolano, M. et al. Small fiber pathology parallels disease progression in Parkinson disease: a longitudinal study. Acta Neuropathol. 136, 501-503 (2018).

23. Kass-lliyya, L. et al. Small fiber neuropathy in Parkinson's disease: a clinical, pathological and corneal confocal microscopy study. Parkinsonism Relat. Disord. 21, 1454-1460 (2015).

24. Bellucci, A., Fiorentini, C., Zaltieri, M., Missale, C. \& Spano, P. The "in situ" proximity ligation assay to probe protein-protein interactions in intact tissues. Methods Mol. Biol. 1174, 397-405 (2014).

25. Roberts, R. F., Wade-Martins, R. \& Alegre-Abarrategui, J. Direct visualization of alpha-synuclein oligomers reveals previously undetected pathology in Parkinson's disease. Brain. Brain 138, 1642-1657 (2015). 
26. Bengoa-Vergniory, N., Roberts, R. F., Wade-Martins, R. \& Alegre-Abarrategui, J. Alpha-synuclein oligomers: a new hope. Acta Neuropathol. 134, 819-838 (2017).

27. Sekiya, $H$. et al. Wide distribution of alpha-synuclein oligomers in multiple system atrophy brain detected by proximity ligation. Acta Neuropathol. 137, 455-466 (2019).

28. Ruffmann, C. et al. Detection of alpha-synuclein conformational variants from gastro-intestinal biopsy tissue as a potential biomarker for Parkinson's disease. Neuropathol. Appl. Neurobiol. 44, 722-736 (2018).

29. Mazzetti, S. et al. alpha-Synuclein oligomers in skin biopsy of idiopathic and monozygotic twin patients with Parkinson's disease. Brain 143, 920-931 (2020).

30. Beach, T. G. et al. Unified staging system for Lewy body disorders: correlation with nigrostriatal degeneration, cognitive impairment and motor dysfunction. Acta Neuropathol. 117, 613-634 (2009).

31. Braak, H., Rub, U., Gai, W. P. \& Del Tredici, K. Idiopathic Parkinson's disease: possible routes by which vulnerable neuronal types may be subject to neuroinvasion by an unknown pathogen. J. Neural Transm. 110, 517-536 (2003).

32. Donadio, V. et al. Skin alpha-synuclein deposits differ in clinical variants of synucleinopathy: an in vivo study. Sci. Rep. 8, 14246 (2018)

33. Peng, C., Trojanowski, J. Q. \& Lee, V. M. Protein transmission in neurodegenerative disease. Nat. Rev. Neurol. 16, 199-212 (2020).

34. Doppler, K. Detection of dermal alpha-synuclein deposits as a biomarker for Parkinson's disease. J. Parkinsons Dis. https://doi.org/10.3233/JPD-202489 (2021).

35. Oueslati, A. Implication of alpha-synuclein phosphorylation at S129 in synucleinopathies: what have we learned in the last decade? J. Parkinsons Dis. 6, 39-51 (2016).

36. Isonaka, R., Gibbons, C. H., Wang, N., Freeman, R. \& Goldstein, D. S. Association of innervation-adjusted alpha-synuclein in arrector pili muscles with cardiac noradrenergic deficiency in autonomic synucleinopathies. Clin. Auton. Res. 29, 587-593 (2019)

37. Donadio, V. et al. In vivo diagnosis of synucleinopathies: a comparative study of skin biopsy and RT-QulC. Neurology 96, e2513-e2524 (2021).

38. Donadio, V. et al. Skin biopsy may help to distinguish multiple system atrophyParkinsonism from Parkinson's disease with orthostatic hypotension. Mov. Disord. 35, 1649-1657 (2020)

39. Che, N. N. \& Yang, H. Q. Potential use of corneal confocal microscopy in the diagnosis of Parkinson's disease associated neuropathy. Transl. Neurodegener. $\mathbf{9}$, 28 (2020).

40. Cossu, G. \& Melis, M. The peripheral nerve involvement in Parkinson disease: a multifaceted phenomenon. Parkinsonism Relat. Disord. 25, 17-20 (2016).

41. Espay, A. J. et al. Disease modification and biomarker development in Parkinson disease: revision or reconstruction? Neurology 94, 481-494 (2020).

42. Lauria, G. et al. EFNS guidelines on the use of skin biopsy in the diagnosis of peripheral neuropathy. Eur. J. Neurol. 12, 747-758 (2005).

43. Lauria, G. et al. European Federation of Neurological Societies/Peripheral Nerve Society Guideline on the use of skin biopsy in the diagnosis of small fiber neuropathy. Report of a joint task force of the European Federation of Neurological Societies and the Peripheral Nerve Society. Eur. J. Neurol. 17, 903-912 (2010). e944-909.

44. Hughes, A. J., Daniel, S. E., Kilford, L. \& Lees, A. J. Accuracy of clinical diagnosis of idiopathic Parkinson's disease: a clinico-pathological study of 100 cases. J. Neurol. Neurosurg. Psychiatry 55, 181-184 (1992).

45. Gilman, S. et al. Second consensus statement on the diagnosis of multiple system atrophy. Neurology 71, 670-676 (2008).

46. Hoglinger, G. U. et al. Clinical diagnosis of progressive supranuclear palsy: the movement disorder society criteria. Mov. Disord. 32, 853-864 (2017).

47. Armstrong, M. J. et al. Criteria for the diagnosis of corticobasal degeneration. Neurology 80, 496-503 (2013)

48. Hoehn, M. M. \& Yahr, M. D. Parkinsonism: onset, progression and mortality. Neurology 17, 427-442 (1967).

49. Fahn, S. \& Elton, R. UPDRS program members. In Recent Developments in Par kinsons Disease Vol. 2 (eds. Fahn, S., Marsden, C. D., Goldstein, M. \& Calne, D. B.) (Macmillan Healthcare Information, 1987).

50. Folstein, M. F., Folstein, S. E. \& McHugh, P. R. "Mini-mental state". A practical method for grading the cognitive state of patients for the clinician. J. Psychiatr Res. 12, 189-198 (1975).

51. Nasreddine, Z. S. et al. The Montreal cognitive assessment, MoCA: a brief screening tool for mild cognitive impairment. J. Am. Geriatr. Soc. 53, 695-699 (2005).

52. Sletten, D. M., Suarez, G. A., Low, P. A., Mandrekar, J. \& Singer, W. COMPASS 31: a refined and abbreviated composite autonomic symptom score. Mayo Clin. Proc. 87, 1196-1201 (2012)
53. Beck, A. T., Steer, R. A. \& Brown, G. K. Manual for the Beck Depression Inventory-II. (San Antonio, TX, Psychological Corporation, (1996).

54. Stiasny-Kolster, K. et al. The REM sleep behavior disorder screening questionnaire - A new diagnostic instrument. Mov. Disord. 22, 2386-2393 (2007).

55. Tomlinson, C. L. et al. Systematic review of levodopa dose equivalency reporting in Parkinson's disease. Mov. Disord. 25, 2649-2653 (2010).

56. Vacchi, E., Pinton, S., Kaelin-Lang, A. \& Melli, G. Targeting alpha synuclein aggregates in cutaneous peripheral nerve fibers by free-floating immunofluorescence assay. J. Vis. Exp. https://doi.org/10.3791/59558 (2019).

57. Provitera, V. et al. A multi-center, multinational age- and gender-adjusted normative dataset for immunofluorescent intraepidermal nerve fiber density at the distal leg. Eur. J. Neurol. 23, 333-338 (2016).

\section{ACKNOWLEDGEMENTS}

The authors are very grateful to all the patients and their relatives who participated in this study. They are grateful to Mrs. Nicole Vago, research nurse, for her valuable work on the clinical database. The research leading to these results received funding from FIDINAM 05.2020.

\section{AUTHOR CONTRIBUTIONS}

E.V.: Data acquisition, analysis and interpretation, and manuscript draft. Camilla Senese: Data acquisition, manuscript revision for intellectual content. G.C.: Data acquisition and interpretation, manuscript revision for intellectual content. G. D.:Data analysis and manuscript revision for intellectual content. S.P.: Data acquisition and manuscript revision for intellectual content. S.M., I.B., G.B., Claudio Staedler, S.G.: Patient enrollment, manuscript revision for intellectual content. C.G.: Data interpretation and manuscript revision for intellectual content. A.K.-L.: Study design, patient enrollment, interpretation of data, and manuscript revision for intellectual content. G.M.: Study design, patient enrollment, data interpretation, manuscript draft, and revision.

\section{COMPETING INTERESTS}

The authors declare no competing interests.

\section{ADDITIONAL INFORMATION}

Supplementary information The online version contains supplementary material available at https://doi.org/10.1038/s41531-021-00262-y.

Correspondence and requests for materials should be addressed to Giorgia Melli.

Reprints and permission information is available at http://www.nature.com/ reprints

Publisher's note Springer Nature remains neutral with regard to jurisdictional claims in published maps and institutional affiliations.

(i) Open Access This article is licensed under a Creative Common Attribution 4.0 International License, which permits use, sharing, adaptation, distribution and reproduction in any medium or format, as long as you give appropriate credit to the original author(s) and the source, provide a link to the Creative Commons license, and indicate if changes were made. The images or other third party material in this article are included in the article's Creative Commons license, unless indicated otherwise in a credit line to the material. If material is not included in the article's Creative Commons license and your intended use is not permitted by statutory regulation or exceeds the permitted use, you will need to obtain permission directly from the copyright holder. To view a copy of this license, visit http://creativecommons. org/licenses/by/4.0/.

(c) The Author(s) 2021 